ANALELE ŞTIINŢIFICE ALE UNIVERSITĂŢII "AL.I. CUZA” DIN IAŞI (S.N.)

MATEMATICĂ, Tomul LX, 2014, f.2

DOI: $10.2478 /$ aicu-2013-0034

\title{
SMALL COVERS OVER THE PRODUCT OF 3-SIDED PRISM WITH $n$-SIMPLEX
}

BY

\author{
YANCHANG CHEN
}

\begin{abstract}
In this paper, the author calculates the number of equivariant homeomorphism classes of (orientable) small covers over the product of 3-sided prism with $n$-simplex.
\end{abstract}

Mathematics Subject Classification 2010: 57S10, 57S25, 52B11.

Key words: small cover, equivariant homeomorphism, polytope.

\section{Introduction}

A small cover, defined by DAVIS and JANUSZKIEWICZ in [9], is a smooth closed manifold $M^{n}$ with a locally standard $\left(\mathbb{Z}_{2}\right)^{n}$-action such that its orbit space is a simple convex polytope. For instance, the real projective space $\mathbb{R} P^{n}$ with a natural $\left(\mathbb{Z}_{2}\right)^{n}$-action is a small cover over an $n$-simplex. This gives a direct connection between equivariant topology and combinatorics and makes it possible to study the topology of small covers through the combinatorial structure of quotient spaces.

In [13], LÜ and MASUDA showed that the equivariant homeomorphism class of a small cover over a simple convex polytope $P^{n}$ agrees with the equivalence class of its corresponding $\left(\mathbb{Z}_{2}\right)^{n}$-coloring under the action of automorphism group of face poset of $P^{n}$. This holds for orientable small covers by the orientability condition in [15] (see Theoerem 4.4). But there aren't general formulas to calculate the number of equivariant homeomorphism classes of (orientable) small covers over an arbitrary simple convex polytope. 
In recent years, several studies have attempted to enumerate the number of equivalence classes of all small covers over a specific polytope. GARRISON and SCOTT [10] used a computer program to calculate the number of homeomorphism classes of all small covers over a dodecahedron. In 2008, CHOI [6] determined the number of equivariant homeomorphism classes of small covers over cubes. Prisms are an interesting class of 3-dimensional polytopes. CAO and LÜ [4] classified small covers over prisms up to homeomorphism and calculated the number of homeomorphism classes of small covers over prisms. CAI, CHEN and LÜ [3] calculated the number of equivariant homeomorphism classes of small covers over prisms. As a generalization, WANG and CHEN [17] determined the number of equivariant homeomorphism classes of small covers over products of a polygon with a simplex. Furthermore, CHEN and WANG [5] calculated the number of D-J equivalence classes of all orientable small covers over products of a prism with a simplex.

The smallest interesting prism is the 3-sided prism. L $\ddot{U}$ and YU [14] showed that one can obtain each 3-dimensional small cover from the small cover $S^{1} \times \mathbb{R} P^{2}$ over the 3 -sided prism and the small cover $\mathbb{R} P^{3}$ over the 3 -simplex by using cut and paste strategies in the sense of six equivariant operations. Let $\widehat{\mathcal{M}}$ be the set of equivariant unoriented cobordism classes of all 3-dimensional small covers. Then $\widehat{\mathcal{M}}$ is generated by the classes of $S^{1} \times \mathbb{R} P^{2}$ over the 3 -sided prism and $\mathbb{R} P^{3}$ over the 3 -simplex $([12,14])$. Much further research on small covers over the 3 -sided prism has been carried on $([11,16])$. An example of a cohomologically non-rigid polytope was obtained from a 3 -sided prism by iterating the operation of vertex cut twice ([8]). Products of polytopes are another interesting class of polytopes and more complicated than one might think ([18]). And small covers over products of simple convex polytopes have become an important search object $([5,6,7$, 17]). Motivated by these, the author calculates the number of equivariant homeomorphism classes of small covers over the product of 3 -sided prism with $n$-simplex in this paper.

From [15], there exist orientable small covers over every simple convex 3 -polytope and there exist non-orientable small covers over every simple convex 3-polytope, except the 3-simplex. An orientable 3-dimensional small cover corresponds to a 4-colored simple convex 3-polytope and the existence of an orientable small cover over every simple convex 3-polytope is closely related to the four color theorem (see [2] for the four color theorem). In [7], CHOI calculated the number of D-J equivalence classes of orientable small covers over cubes. The number of equivariant homeomorphism classes of 
orientable small covers over the product of 3 -sided prism with $n$-simplex is also calculated in this paper.

Let $P_{3}, \Delta^{n}$ be a 3 -sided prism (i.e., the product of a 3 -gon and the interval $I$ ) and an $n$-simplex respectively. The main results of this paper are stated as follows:

Theorem 1.1. Let $E\left(P_{3} \times \Delta^{n}\right)$ be the number of equivariant homeomorphism classes of small covers over $P_{3} \times \Delta^{n}$. When $n \geq 3$,

$$
E\left(P_{3} \times \Delta^{n}\right)=\frac{\left(5 \cdot 4^{n}+9 \cdot 2^{n+1}+33\right) \prod_{k=1}^{n+3}\left(2^{n+3}-2^{k-1}\right)}{12(n+1) !} .
$$

Up to equivariant homeomorphism, there are 12846400 small covers over $P_{3} \times \Delta_{2}, 47460$ small covers over $P_{3} \times I$ and 98 small covers over $P_{3}$.

Theorem 1.2. Let $E_{o}\left(P_{3} \times \Delta^{n}\right)$ be the number of equivariant homeomorphism classes of orientable small covers over $P_{3} \times \Delta^{n}$. When $n \geq 3$ and $n$ is odd,

$$
E_{o}\left(P_{3} \times \Delta^{n}\right)=\frac{\left(5 \cdot 4^{n-1}+2^{n}+6\right) \prod_{k=1}^{n+3}\left(2^{n+3}-2^{k-1}\right)}{12(n+1) !} .
$$

When $n \geq 4$ and $n$ is even,

$$
E_{o}\left(P_{3} \times \Delta^{n}\right)=\frac{\left(2^{n+1}+6\right) \prod_{k=1}^{n+3}\left(2^{n+3}-2^{k-1}\right)}{12(n+1) !} .
$$

Up to equivariant homeomorphism, there are 972160 orientable small covers over $P_{3} \times \Delta_{2}, 8400$ orientable small covers over $P_{3} \times I$ and 28 orientable small covers over $P_{3}$.

The paper is organized as follows. In Section 2, we review the basic theory about small covers and calculate the automorphism group of face poset of $P_{3} \times \Delta^{n}$. In Section 3, we determine the number of all $\left(\mathbb{Z}_{2}\right)^{n+3}$ colorings on $P_{3} \times \Delta^{n}$ and prove Theorem 1.1. In Section 4 , we calculate the number of all orientable colorings on $P_{3} \times \Delta^{n}$ and prove Theorem 1.2.

\section{Preliminaries}

A convex polytope $P^{n}$ of dimension $n$ is said to be simple if every vertex of $P^{n}$ is the intersection of exactly $n$ facets (i.e. faces of dimension $(n-1)$ ) 
(see [18]). An $n$-dimensional smooth closed manifold $M^{n}$ is said to be a small cover if it admits a smooth $\left(\mathbb{Z}_{2}\right)^{n}$-action such that the action is locally isomorphic to a standard action of $\left(\mathbb{Z}_{2}\right)^{n}$ on $\mathbb{R}^{n}$ and the orbit space $M^{n} /\left(\mathbb{Z}_{2}\right)^{n}$ is a simple convex polytope of dimension $n$.

Let $P^{n}$ be a simple convex polytope of dimension $n$ and $\mathcal{F}\left(P^{n}\right)=$ $\left\{F_{1}, \ldots, F_{\ell}\right\}$ be the set of facets of $P^{n}$. Suppose that $\pi: M^{n} \rightarrow P^{n}$ is a small cover over $P^{n}$. Then there are $\ell$ connected submanifolds $\pi^{-1}\left(F_{1}\right), \ldots, \pi^{-1}\left(F_{\ell}\right)$. Each submanifold $\pi^{-1}\left(F_{i}\right)$ is fixed pointwise by a $\mathbb{Z}_{2}-\operatorname{subgroup} \mathbb{Z}_{2}\left(F_{i}\right)$ of $\left(\mathbb{Z}_{2}\right)^{n}$, so that each facet $F_{i}$ corresponds to the $\mathbb{Z}_{2}$-subgroup $\mathbb{Z}_{2}\left(F_{i}\right)$. Obviously, the $\mathbb{Z}_{2}$-subgroup $\mathbb{Z}_{2}\left(F_{i}\right)$ actually agrees with an element $\nu_{i}$ in $\left(\mathbb{Z}_{2}\right)^{n}$ as a vector space. For each face $F$ of codimension $u$, since $P^{n}$ is simple, there are $u$ facets $F_{i_{1}}, \ldots, F_{i_{u}}$ such that $F=F_{i_{1}} \cap \ldots \cap F_{i_{u}}$. Then, the corresponding submanifolds $\pi^{-1}\left(F_{i_{1}}\right), \ldots, \pi^{-1}\left(F_{i_{u}}\right)$ intersect transversally in the $(n-u)$-dimensional submanifold $\pi^{-1}(F)$, and the isotropy subgroup $\mathbb{Z}_{2}(F)$ of $\pi^{-1}(F)$ is a subtorus of rank $u$ and is generated by $\mathbb{Z}_{2}\left(F_{i_{1}}\right), \ldots, \mathbb{Z}_{2}\left(F_{i_{u}}\right)$ (or is determined by $\nu_{i_{1}}, \ldots, \nu_{i_{u}}$ in $\left(\mathbb{Z}_{2}\right)^{n}$ ) (see [9]).

Consider a map $\lambda: \mathcal{F}\left(P^{n}\right) \longrightarrow\left(\mathbb{Z}_{2}\right)^{n}$ which satisfies the nonsingularity condition: $\left\{\lambda\left(F_{i_{1}}\right), \ldots, \lambda\left(F_{i_{n}}\right)\right\}$ is a basis of $\left(\mathbb{Z}_{2}\right)^{n}$ whenever the intersection $F_{i_{1}} \cap \ldots \cap F_{i_{n}}$ is non-empty. We call $\lambda$ a characteristic function. If we regard each nonzero vector of $\left(\mathbb{Z}_{2}\right)^{n}$ as being a color, then the characteristic function $\lambda$ means that each facet is colored by a color. Here we also call $\lambda$ a $\left(\mathbb{Z}_{2}\right)^{n}$-coloring on $P^{n}$.

In fact, DAVIS and JANUSZKIEWICZ [9] gave a reconstruction process of a small cover by using a $\left(\mathbb{Z}_{2}\right)^{n}$-coloring $\lambda: \mathcal{F}\left(P^{n}\right) \longrightarrow\left(\mathbb{Z}_{2}\right)^{n}$. Let $\mathbb{Z}_{2}\left(F_{i}\right)$ be the subgroup of $\left(\mathbb{Z}_{2}\right)^{n}$ generated by $\lambda\left(F_{i}\right)$. Given a point $p \in P^{n}$, by $F(p)$ we denote the minimal face containing $p$ in its relative interior. Assume $F(p)=F_{i_{1}} \cap \ldots \cap F_{i_{u}}$ and $\mathbb{Z}_{2}(F(p))=\bigoplus_{j=1}^{u} \mathbb{Z}_{2}\left(F_{i_{j}}\right)$. Note that $\mathbb{Z}_{2}(F(p))$ is a $u$-dimensional subgroup of $\left(\mathbb{Z}_{2}\right)^{n}$. Let $M(\lambda)$ denote $P^{n} \times\left(\mathbb{Z}_{2}\right)^{n} / \sim$, where $(p, g) \sim(q, h)$ if $p=q$ and $g^{-1} h \in \mathbb{Z}_{2}(F(p))$. The free action of $\left(\mathbb{Z}_{2}\right)^{n}$ on $P^{n} \times\left(\mathbb{Z}_{2}\right)^{n}$ descends to an action on $M(\lambda)$ with quotient $P^{n}$. Thus $M(\lambda)$ is a small cover over $P^{n}$.

Two small covers $M_{1}$ and $M_{2}$ over $P^{n}$ are said to be weakly equivariantly homeomorphic if there is an automorphism $\varphi:\left(\mathbb{Z}_{2}\right)^{n} \rightarrow\left(\mathbb{Z}_{2}\right)^{n}$ and a homeomorphism $f: M_{1} \rightarrow M_{2}$ such that $f(t \cdot x)=\varphi(t) \cdot f(x)$ for every $t \in\left(\mathbb{Z}_{2}\right)^{n}$ and $x \in M_{1}$. If $\varphi$ is an identity, then $M_{1}$ and $M_{2}$ are equivariantly homeomorphic. Following [9], two small covers $M_{1}$ and $M_{2}$ over $P^{n}$ are said to be Davis-Januszkiewicz equivalent (or simply, D-J equivalent) if there is a weakly equivariant homeomorphism $f: M_{1} \rightarrow M_{2}$ covering the identity 
on $P^{n}$.

By $\Lambda\left(P^{n}\right)$ we denote the set of all $\left(\mathbb{Z}_{2}\right)^{n}$-colorings on $P^{n}$. Then we have:

Theorem 2.1. All small covers over $P^{n}$ are given by $\left\{M(\lambda) \mid \lambda \in \Lambda\left(P^{n}\right)\right\}$, i.e. for each small cover $M^{n}$ over $P^{n}$, there is a $\left(\mathbb{Z}_{2}\right)^{n}$-coloring $\lambda$ with an equivariant homeomorphism $M(\lambda) \longrightarrow M^{n}$ covering the identity on $P^{n}$.

Remark 2.2. Generally speaking, we can't make sure that there always exist small covers over a simple convex polytope $P^{n}$ when $n \geq 4$. For example, see Nonexample 1.22 of [9]. However, the Four Color Theorem makes sure that there always exist small covers over every 3-dimensional simple convex polytope, so there exist small covers over $P_{3}$. Since $\mathbb{R} P^{n}$ is a small cover over $\Delta^{n}$, there exist small covers over $P_{3} \times \Delta^{n}$.

There is a natural action of $G L\left(n, \mathbb{Z}_{2}\right)$ on $\Lambda\left(P^{n}\right)$ defined by the correspondence $\lambda \longmapsto \sigma \circ \lambda$, and the action on $\Lambda\left(P^{n}\right)$ is free. Without loss of generality, we assume that $F_{1}, \ldots, F_{n}$ of $\mathcal{F}\left(P^{n}\right)$ meet at one vertex $p$ of $P^{n}$. Let $e_{1}, \ldots, e_{n}$ be the standard basis of $\left(\mathbb{Z}_{2}\right)^{n}$. Write $A\left(P^{n}\right)=\{\lambda \in$ $\Lambda\left(P^{n}\right) \mid \lambda\left(F_{i}\right)=e_{i}$ for $\left.i=1, \ldots, n\right\}$. In fact, $A\left(P^{n}\right)$ is the orbit space of $\Lambda\left(P^{n}\right)$ under the action of $G L\left(n, \mathbb{Z}_{2}\right)$. Then we have

Lemma 2.3. $\left|\Lambda\left(P^{n}\right)\right|=\left|A\left(P^{n}\right)\right| \times\left|G L\left(n, \mathbb{Z}_{2}\right)\right|$.

Note that $\left|G L\left(n, \mathbb{Z}_{2}\right)\right|=\prod_{k=1}^{n}\left(2^{n}-2^{k-1}\right) \quad([1])$. Two small covers $M\left(\lambda_{1}\right)$ and $M\left(\lambda_{2}\right)$ over $P^{n}$ are D-J equivalent if and only if there is $\sigma \in$ $G L\left(n, \mathbb{Z}_{2}\right)$ such that $\lambda_{1}=\sigma \circ \lambda_{2}$. So the number of D-J equivalence classes of small covers over $P^{n}$ is $\left|A\left(P^{n}\right)\right|$.

Let $P^{n}$ be a simple convex polytope of dimension $n$. All faces of $P^{n}$ form a poset (i.e. a partially ordered set by inclusion). An automorphism of $\mathcal{F}\left(P^{n}\right)$ is a bijection from $\mathcal{F}\left(P^{n}\right)$ to itself which preserves the poset structure of all faces of $P^{n}$, and by $\operatorname{Aut}\left(\mathcal{F}\left(P^{n}\right)\right)$ we denote the group of automorphisms of $\mathcal{F}\left(P^{n}\right)$. One can define the right action of $\operatorname{Aut}\left(\mathcal{F}\left(P^{n}\right)\right)$ on $\Lambda\left(P^{n}\right)$ by $\lambda \times h \longmapsto \lambda \circ h$, where $\lambda \in \Lambda\left(P^{n}\right)$ and $h \in \operatorname{Aut}\left(\mathcal{F}\left(P^{n}\right)\right)$. The following theorem is well known (see [13]).

Theorem 2.4. Two small covers over an $n$-dimensional simple convex polytope $P^{n}$ are equivariantly homeomorphic if and only if there is $h \in$ Aut $\left(\mathcal{F}\left(P^{n}\right)\right)$ such that $\lambda_{1}=\lambda_{2} \circ h$, where $\lambda_{1}$ and $\lambda_{2}$ are their corresponding $\left(\mathbb{Z}_{2}\right)^{n}$-colorings on $P^{n}$. 
So the number of orbits of $\Lambda\left(P^{n}\right)$ under the action of $\operatorname{Aut}\left(\mathcal{F}\left(P^{n}\right)\right)$ is just the number of equivariant homeomorphism classes of small covers over $P^{n}$. Thus, we are going to count the orbits. Burnside Lemma is very useful in the enumeration of the number of orbits.

Burnside Lemma. Let $G$ be a finite group acting on a set $X$. Then the number of orbits of $X$ under the action of $G$ equals $\frac{1}{|G|} \sum_{g \in G}\left|X_{g}\right|$, where $X_{g}=\{x \in X \mid g x=x\}$.

Burnside Lemma suggests that we need to understand the structure of $\operatorname{Aut}\left(\mathcal{F}\left(P^{n}\right)\right)$ in order to determine the number of the orbits of $\Lambda\left(P^{n}\right)$ under the action of $\operatorname{Aut}\left(\mathcal{F}\left(P^{n}\right)\right)$. We shall particularly be concerned with the case in which the simple convex polytope is $P_{3} \times \Delta^{n}$.

To be convenient, we introduce the following marks. By $s_{1}^{\prime}$ and $s_{2}^{\prime}$ we denote the top and bottom facets of $P_{3}$ respectively, and by $a_{1}^{\prime}, a_{2}^{\prime}, a_{3}^{\prime}$ we denote three sided facets of $P_{3}$ in their general order. For $n$-simplex $\Delta^{n}$, by $b_{1}^{\prime}, b_{2}^{\prime}, \ldots, b_{n+1}^{\prime}$ we denote all facets of $\Delta^{n}$. Set $\mathcal{F}^{\prime}=\left\{s_{1}=s_{1}^{\prime} \times \Delta^{n}, s_{2}=\right.$ $\left.s_{2}^{\prime} \times \Delta^{n}, a_{i}=a_{i}^{\prime} \times \Delta^{n} \mid 1 \leq i \leq 3\right\}, \mathcal{F}^{\prime \prime}=\left\{b_{j}=P_{3} \times b_{j}^{\prime} \mid 1 \leq j \leq n+1\right\}$. Then $\mathcal{F}\left(P_{3} \times \Delta^{n}\right)=\mathcal{F}^{\prime} \cup \mathcal{F}^{\prime \prime}$.

Lemma 2.5. Let $P_{3}, \Delta^{n}$ be a 3-sided prism and an n-simplex respectively. The automorphism group $\operatorname{Aut}\left(\mathcal{F}\left(P_{3} \times \Delta^{n}\right)\right)$ is isomorphic to

$$
\begin{cases}S_{3} \times\left(\mathbb{Z}_{2}\right)^{3}, & n=1, \\ \left(S_{3}\right)^{2} \times\left(\mathbb{Z}_{2}\right)^{2}, & n=2, \\ S_{3} \times \mathbb{Z}_{2} \times S_{n+1}, & n \geq 3\end{cases}
$$

where $S_{n+1}$ is the symmetric group of rank $n+1$.

Proof. First, we show that the automorphism group $\operatorname{Aut}\left(\mathcal{F}\left(P_{3}\right)\right)$ is isomorphic to $S_{3} \times \mathbb{Z}_{2}$. Let three sided facets of $P_{3}$ interchange and the top and bottom facets stay unchanged. Then these automorphisms form a group $S_{3}$. Let the top and bottom facets interchange and three sided facets of $P_{3}$ stay unchanged. These automorphisms form a group $\mathbb{Z}_{2}$. Since any one of all sided facets cannot be mapped to the top facet or bottom facet under the automorphisms of $\mathcal{F}\left(P_{3}\right), \operatorname{Aut}\left(\mathcal{F}\left(P_{3}\right)\right)$ is isomorphic to $S_{3} \times \mathbb{Z}_{2}$.

When $n \geq 3$, the facets of $\mathcal{F}^{\prime}$ and $\mathcal{F}^{\prime \prime}$ are mapped to $\mathcal{F}^{\prime}$ and $\mathcal{F}^{\prime \prime}$ respectively under the automorphisms of $\operatorname{Aut}\left(\mathcal{F}\left(P_{3} \times \Delta^{n}\right)\right)$. Since $\operatorname{Aut}\left(\mathcal{F}\left(P_{3}\right)\right)$ is isomorphic to $S_{3} \times \mathbb{Z}_{2}$ and $\operatorname{Aut}\left(\mathcal{F}\left(\Delta^{n}\right)\right)$ is isomorphic to $S_{n+1}$, Aut ( $\left.\mathcal{F}\left(P_{3} \times \Delta^{n}\right)\right)$ is isomorphic to $S_{3} \times \mathbb{Z}_{2} \times S_{n+1}$. 
Next, we consider the case in which $n=2$. Below we show that the automorphism group $\operatorname{Aut}\left(\mathcal{F}\left(\Delta^{2} \times \Delta^{2}\right)\right)$ is isomorphic to $S_{3} \times S_{3} \times \mathbb{Z}_{2}$. $\Delta^{2}$ is a 3 -gon. By $c_{1}^{\prime}, c_{2}^{\prime}, c_{3}^{\prime}$ we denote three edges of $\Delta^{2}$ in their general order. Let $\mathcal{F}_{1}^{\prime}=\left\{c_{i}=c_{i}^{\prime} \times \Delta^{2} \mid 1 \leq i \leq 3\right\}, \mathcal{F}_{1}^{\prime \prime}=\left\{d_{j}=\Delta^{2} \times c_{j}^{\prime} \mid 1 \leq j \leq 3\right\}$. Then $\mathcal{F}\left(\Delta^{2} \times \Delta^{2}\right)=\mathcal{F}_{1}^{\prime} \cup \mathcal{F}_{1}^{\prime \prime}$. There are automorphisms of $\mathcal{F}\left(\Delta^{2} \times \Delta^{2}\right)$ under which the facets of $\mathcal{F}_{1}^{\prime}$ and $\mathcal{F}_{1}^{\prime \prime}$ are mapped to $\mathcal{F}_{1}^{\prime}$ and $\mathcal{F}_{1}^{\prime \prime}$ respectively. These automorphisms form a group $S_{3} \times S_{3}$. We choose an automorphism $f$ such that $f\left(c_{i}\right)=d_{i}$ for $1 \leq i \leq 3$ and $f\left(d_{j}\right)=c_{j}$ for $1 \leq j \leq 3$. Let $\mathbb{Z}_{2}=\{f, 1\}$. Then we get a new group $S_{3} \times S_{3} \times \mathbb{Z}_{2}$, each of which is an automorphism under which the facets in $\mathcal{F}_{1}^{\prime}$ and $\mathcal{F}_{1}^{\prime \prime}$ are mapped to $\mathcal{F}_{1}^{\prime}$ and $\mathcal{F}_{1}^{\prime \prime}$ or to $\mathcal{F}_{1}^{\prime \prime}$ and $\mathcal{F}_{1}^{\prime}$ respectively. Since other bijections from $\mathcal{F}\left(\Delta^{2} \times \Delta^{2}\right)$ to itself don't preserve the poset structure of all faces of $\Delta^{2} \times \Delta^{2}, \operatorname{Aut}\left(\mathcal{F}\left(\Delta^{2} \times \Delta^{2}\right)\right)$ is isomorphic to $S_{3} \times S_{3} \times \mathbb{Z}_{2}$.

When $n=2, P_{3} \times \Delta^{n}$ is $\Delta^{2} \times I \times \Delta^{2}$. Since $\operatorname{Aut}\left(\mathcal{F}\left(\Delta^{2} \times \Delta^{2}\right)\right)$ is isomorphic to $S_{3} \times S_{3} \times \mathbb{Z}_{2}$ and $\operatorname{Aut}(\mathcal{F}(I))$ is isomorphic to $\mathbb{Z}_{2}, \operatorname{Aut}\left(\mathcal{F}\left(P_{3} \times \Delta^{2}\right)\right)$ is isomorphic to $S_{3} \times S_{3} \times \mathbb{Z}_{2} \times \mathbb{Z}_{2}$.

Finally, we consider the case in which $n=1$. Below we show that the automorphism group $\operatorname{Aut}\left(\mathcal{F}\left(I^{2}\right)\right)$ is isomorphic to $\left(\mathbb{Z}_{2}\right)^{3}$. Aut $\left(\mathcal{F}\left(I^{2}\right)\right)$ contains a $\mathbb{Z}_{2}$-subgroup since there is one automorphism for the permutation of the two pairs of opposite facets of $I^{2}=I \times I$. All elements of $\operatorname{Aut}\left(\mathcal{F}\left(I^{2}\right)\right)$ can be written in a simple form as follows: $a^{e_{1}} b^{e_{2}} \cdot u$, where $e_{1}, e_{2} \in \mathbb{Z}_{2}$, with reflections $a, b$ and $u \in$ the former $\mathbb{Z}_{2}$-subgroup. Thus, $\operatorname{Aut}\left(\mathcal{F}\left(I^{2}\right)\right)$ is isomorphic to $\left(\mathbb{Z}_{2}\right)^{3}$.

When $n=1, P_{3} \times \Delta^{n}$ is $\Delta^{2} \times I^{2}$. Since the automorphism group $\operatorname{Aut}\left(\mathcal{F}\left(\Delta^{2}\right)\right)$ is isomorphic to $S_{3}$ and $\operatorname{Aut}\left(\mathcal{F}\left(I^{2}\right)\right)$ is isomorphic to $\left(\mathbb{Z}_{2}\right)^{3}$, $\operatorname{Aut}\left(\mathcal{F}\left(P_{3} \times I\right)\right)$ is isomorphic to $S_{3} \times\left(\mathbb{Z}_{2}\right)^{3}$.

\section{The number of small covers over $P_{3} \times \Delta^{n}$}

First, we give a criterion for a map $\lambda: \mathcal{F}\left(P_{3} \times \Delta^{n}\right) \longrightarrow\left(\mathbb{Z}_{2}\right)^{n+3}$ to be a characteristic function. The nonsingularity condition of the characteristic function means the following:

(1) $\left\{\lambda\left(s_{1}\right), \lambda\left(a_{1}\right), \lambda\left(a_{2}\right), \lambda\left(b_{1}\right), \ldots, \lambda\left(b_{n}\right)\right\}$ is a basis of $\left(\mathbb{Z}_{2}\right)^{n+3}$.

(2) $\lambda\left(b_{n+1}\right)$ satisfies that $\left\{\lambda\left(b_{n+1}\right), \lambda\left(b_{k_{1}}\right), \ldots, \lambda\left(b_{k_{n-1}}\right), \lambda\left(s_{1}\right), \lambda\left(a_{1}\right), \lambda\left(a_{2}\right)\right\}$ is a basis of $\left(\mathbb{Z}_{2}\right)^{n+3}$, where $k_{1}<k_{2}<\ldots<k_{n-1}$ and $k_{1}, \ldots, k_{n-1} \in$ $\{1,2, \ldots, n\}$. 
(3) $\lambda\left(a_{3}\right)$ satisfies that $\left\{\lambda\left(a_{3}\right), \lambda\left(s_{1}\right), \lambda\left(a_{l_{1}}\right), \lambda\left(b_{h_{1}}\right), \ldots, \lambda\left(b_{h_{n}}\right)\right\}$ is a basis of $\left(\mathbb{Z}_{2}\right)^{n+3}$, where $l_{1} \in\{1,2\}, \quad h_{1}<h_{2}<\ldots<h_{n}$ and $h_{1}, \ldots, h_{n} \in$ $\{1,2, \ldots, n+1\}$.

(4) $\lambda\left(s_{2}\right)$ satisfies that $\left\{\lambda\left(s_{2}\right), \lambda\left(a_{j_{1}}\right), \lambda\left(a_{j_{2}}\right), \lambda\left(b_{h_{1}}\right), \ldots, \lambda\left(b_{h_{n}}\right)\right\}$ is a basis of $\left(\mathbb{Z}_{2}\right)^{n+3}$, where $j_{1}<j_{2}, j_{1}, j_{2} \in\{1,2,3\}, h_{1}<h_{2}<\ldots<h_{n}$ and $h_{1}, \ldots, h_{n} \in\{1,2, \ldots, n+1\}$.

Next, we calculate the number of $\left(\mathbb{Z}_{2}\right)^{n+3}$-colorings on $P_{3} \times \Delta^{n}$.

Lemma 3.1. When $n \geq 1$, the number of $\left(\mathbb{Z}_{2}\right)^{n+3}$-colorings over $P_{3} \times$ $\Delta^{n}$ is $\left|\Lambda\left(P_{3} \times \Delta^{n}\right)\right|=\left(5 \cdot 4^{n}+7 \cdot 2^{n+1}+21\right) \prod_{k=1}^{n+3}\left(2^{n+3}-2^{k-1}\right)$.

Proof. Let $e_{1}, e_{2}, \ldots, e_{n+3}$ be the standard basis of $\left(\mathbb{Z}_{2}\right)^{n+3}$, then $\left(\mathbb{Z}_{2}\right)^{n+3}$ contains $2^{n+3}-1$ nonzero elements (or $2^{n+3}-1$ colors). We choose $s_{1}, a_{1}, a_{2}$ from $\mathcal{F}^{\prime}$ and $b_{1}, \ldots, b_{n}$ from $\mathcal{F}^{\prime \prime}$, then $s_{1}, a_{1}, a_{2}, b_{1}, \ldots, b_{n}$ meet at one vertex of $P_{3} \times \Delta^{n}$. Then

$$
\begin{aligned}
A\left(P_{3} \times \Delta^{n}\right) & =\left\{\lambda \in \Lambda\left(P_{3} \times \Delta^{n}\right) \mid \lambda\left(s_{1}\right)=e_{1}, \lambda\left(a_{1}\right)=e_{2}, \lambda\left(a_{2}\right)\right. \\
& \left.=e_{3}, \lambda\left(b_{i}\right)=e_{i+3}, 1 \leq i \leq n\right\} .
\end{aligned}
$$

Then, by Lemma 2.3, we have that

$$
\left|\Lambda\left(P_{3} \times \Delta^{n}\right)\right|=\left|A\left(P_{3} \times \Delta^{n}\right)\right| \times\left|G L\left(n+3, \mathbb{Z}_{2}\right)\right|=\prod_{k=1}^{n+3}\left(2^{n+3}-2^{k-1}\right)\left|A\left(P_{3} \times \Delta^{n}\right)\right| .
$$

Write

$$
\begin{aligned}
& A_{0}\left(P_{3} \times \Delta^{n}\right)=\left\{\lambda \in A\left(P_{3} \times \Delta^{n}\right) \mid \lambda\left(b_{n+1}\right)=e_{4}+\ldots+e_{n+3}\right\} \\
& A_{1}\left(P_{3} \times \Delta^{n}\right)=\left\{\lambda \in A\left(P_{3} \times \Delta^{n}\right) \mid \lambda\left(b_{n+1}\right)=e_{4}+\ldots+e_{n+3}+e_{h_{1}},\right. \\
& \left.1 \leq h_{1} \leq 3\right\} \\
& A_{2}\left(P_{3} \times \Delta^{n}\right)=\left\{\lambda \in A\left(P_{3} \times \Delta^{n}\right) \mid \lambda\left(b_{n+1}\right)=e_{4}+\ldots+e_{n+3}+e_{k_{1}}+e_{k_{2}},\right. \\
& \left.\quad 1 \leq k_{1}<k_{2} \leq 3\right\} \\
& A_{3}\left(P_{3} \times \Delta^{n}\right)=\left\{\lambda \in A\left(P_{3} \times \Delta^{n}\right) \mid \lambda\left(b_{n+1}\right)=e_{4}+\ldots+e_{n+3}+e_{1}+e_{2}+e_{3}\right\} .
\end{aligned}
$$

By the definition of $\left(\mathbb{Z}_{2}\right)^{n+3}$-colorings, we have that

$$
\left|A\left(P_{3} \times \Delta^{n}\right)\right|=\sum_{i=0}^{3}\left|A_{i}\left(P_{3} \times \Delta^{n}\right)\right| .
$$


Then, our argument proceeds as follows:

(I) Calculation of $\left|A_{0}\left(P_{3} \times \Delta^{n}\right)\right|$.

In this case, $\lambda\left(a_{3}\right)=e_{2}+e_{3}+e_{m_{1}} \ldots+e_{m_{l}}$, where $1 \leq m_{1}<\ldots<$ $m_{l} \leq n+3, m_{1} \neq 2, m_{1} \neq 3, \ldots, m_{l} \neq 2, m_{l} \neq 3$ and $0 \leq l \leq n+1$. When $\lambda\left(a_{3}\right)=e_{2}+e_{3}+e_{m_{1}}+\ldots+e_{m_{l}}, 4 \leq m_{1}<\ldots<m_{l} \leq n+3$ and $0 \leq l \leq n$, $\lambda\left(s_{2}\right)=e_{1}+e_{n_{1}}+\ldots+e_{n_{j}}$, where $2 \leq n_{1}<\ldots<n_{j} \leq n+3$ and $0 \leq j \leq$ $n+2$. When $\lambda\left(a_{3}\right)=e_{2}+e_{3}+e_{1}+e_{m_{1}}+\ldots+e_{m_{l}}, 4 \leq m_{1}<\ldots<m_{l} \leq n+3$ and $0 \leq l \leq n, \lambda\left(s_{2}\right)=e_{1}+e_{g_{1}}+\ldots+e_{g_{k}}$, where $4 \leq g_{1}<\ldots<g_{k} \leq n+3$ and $0 \leq k \leq n$.

Thus, we have $\left|A_{0}\left(P_{3} \times \Delta_{n}\right)\right|=5 \cdot 4^{n}$.

(II) Calculation of $\left|A_{1}\left(P_{3} \times \Delta^{n}\right)\right|$.

The argument is divided into two cases.

Case 1. $\lambda\left(b_{n+1}\right)=e_{4}+\ldots+e_{n+3}+e_{1}$

In this case, $\lambda\left(a_{3}\right)=e_{2}+e_{3}+e_{m_{1}} \ldots+e_{m_{l}}$, where $1 \leq m_{1}<\ldots<$ $m_{l} \leq n+3, m_{1} \neq 2, m_{1} \neq 3, \ldots, m_{l} \neq 2, m_{l} \neq 3$ and $0 \leq l \leq n+1$. When $\lambda\left(a_{3}\right)=e_{2}+e_{3}, \lambda\left(s_{2}\right)=e_{1}, e_{1}+e_{2}, e_{1}+e_{3}, e_{1}+e_{2}+e_{3}$. When $\lambda\left(a_{3}\right)=e_{2}+e_{3}+e_{m_{1}} \ldots+e_{m_{l}}, 1 \leq m_{1}<\ldots<m_{l} \leq n+3, m_{1} \neq 2, m_{1} \neq$ $3, \ldots, m_{l} \neq 2, m_{l} \neq 3$ and $1 \leq l \leq n+1, \lambda\left(s_{2}\right)=e_{1}$.

Case 2. $\lambda\left(b_{n+1}\right)=e_{4}+\ldots+e_{n+3}+e_{2}$ or $e_{4}+\ldots+e_{n+3}+e_{3}$

In this case, no matter which value of $\lambda\left(b_{n+1}\right)$ is chosen, we have $\lambda\left(a_{3}\right)=$ $e_{2}+e_{3}, e_{2}+e_{3}+e_{1}$. When $\lambda\left(a_{3}\right)=e_{2}+e_{3}, \lambda\left(s_{2}\right)=e_{1}+e_{n_{1}}+\ldots+e_{n_{j}}, 2 \leq$ $n_{1}<\ldots<n_{j} \leq n+3$ and $0 \leq j \leq n+2$. When $\lambda\left(a_{3}\right)=e_{2}+e_{3}+e_{1}$, $\lambda\left(s_{2}\right)=e_{1}$. Thus, we have $\left|A_{1}\left(P_{3} \times \Delta_{n}\right)\right|=5 \cdot 2^{n+1}+5$.

(III) Calculation of $\left|A_{2}\left(P_{3} \times \Delta^{n}\right)\right|$.

The argument is also divided into two cases.

Case 1. $\lambda\left(b_{n+1}\right)=e_{4}+\ldots+e_{n+3}+e_{1}+e_{2}$ or $e_{4}+\ldots+e_{n+3}+e_{1}+e_{3}$

In this case, no matter which value of $\lambda\left(b_{n+1}\right)$ is chosen, we have $\lambda\left(a_{3}\right)=$ $e_{2}+e_{3}, e_{2}+e_{3}+e_{1}$. When $\lambda\left(a_{3}\right)=e_{2}+e_{3}, \lambda\left(s_{2}\right)=e_{1}, e_{1}+e_{2}, e_{1}+e_{3}, e_{1}+$ $e_{2}+e_{3}$. When $\lambda\left(a_{3}\right)=e_{2}+e_{3}+e_{1}, \lambda\left(s_{2}\right)=e_{1}$.

Case 2. $\lambda\left(b_{n+1}\right)=e_{4}+\ldots+e_{n+3}+e_{2}+e_{3}$

In this case, $\lambda\left(a_{3}\right)=e_{2}+e_{3}, e_{2}+e_{3}+e_{1}$. When $\lambda\left(a_{3}\right)=e_{2}+e_{3}, \lambda\left(s_{2}\right)=$ $e_{1}+e_{n_{1}}+\ldots+e_{n_{j}}, 2 \leq n_{1}<\ldots<n_{j} \leq n+3$ and $0 \leq j \leq n+2$. When $\lambda\left(a_{3}\right)=e_{2}+e_{3}+e_{1}, \lambda\left(s_{2}\right)=e_{1}$. Thus, we have $\left|A_{2}\left(P_{3} \times \Delta_{n}\right)\right|=2^{n+2}+11$.

(IV) Calculation of $\left|A_{3}\left(P_{3} \times \Delta^{n}\right)\right|$. 
In this case, $\lambda\left(a_{3}\right)=e_{2}+e_{3}, e_{2}+e_{3}+e_{1}$.

When $\lambda\left(a_{3}\right)=e_{2}+e_{3}, \lambda\left(s_{2}\right)=e_{1}, e_{1}+e_{2}, e_{1}+e_{3}, e_{1}+e_{2}+e_{3}$. When $\lambda\left(a_{3}\right)=e_{2}+e_{3}+e_{1}, \lambda\left(s_{2}\right)=e_{1}$. Thus, we have $\left|A_{3}\left(P_{3} \times \Delta_{n}\right)\right|=5$.

Remark 3.2. From Lemma 3.1, the number of D-J equivalence classes of small covers over $P_{3} \times \Delta^{n}$ is $5 \cdot 4^{n}+7 \cdot 2^{n+1}+21$.

The proof of Theorem 1.1. When $n \geq 3$, from Theorem 2.4, Burnside Lemma and Lemma 2.5, we have that

$$
E\left(P_{3} \times \Delta^{n}\right)=\frac{1}{12(n+1) !} \sum_{g \in \operatorname{Aut}\left(\mathcal{F}\left(P_{3} \times \Delta^{n}\right)\right)}\left|\Lambda_{g}\right|,
$$

where $\Lambda_{g}=\left\{\lambda \in \Lambda\left(P_{3} \times \Delta^{n}\right) \mid \lambda=\lambda \circ g\right\}$. When $n \geq 3$, the automorphism group $\operatorname{Aut}\left(\mathcal{F}\left(P_{3} \times \Delta^{n}\right)\right)$ is isomorphic to $S_{3} \times \mathbb{Z}_{2} \times S_{n+1}$. If $g$ is the generator of the $\mathbb{Z}_{2}$-subgroup of $\operatorname{Aut}\left(\mathcal{F}\left(P_{3} \times \Delta^{n}\right)\right)$ and $\lambda \in \Lambda_{g}$, then $\lambda\left(s_{1}\right)=\lambda\left(s_{2}\right)$. By the argument of Lemma 3.1, we have $\left|\Lambda_{g}\right|=\left(2^{n+2}+12\right) \prod_{k=1}^{n+3}\left(2^{n+3}-2^{k-1}\right)$. If $g$ is other automorphism and isn't unit element of $\operatorname{Aut}\left(\mathcal{F}\left(P_{3} \times \Delta^{n}\right)\right)$, by the nonsingularity condition of characteristic functions, we have that $\Lambda_{g}$ is empty. Thus, from Lemma 3.1, when $n \geq 3$,

$$
\begin{aligned}
& E\left(P_{3} \times \Delta^{n}\right)= \frac{1}{12(n+1) !}\left(2^{n+2}+12+5 \cdot 4^{n}+7 \cdot 2^{n+1}+21\right) . \\
& \cdot \prod_{k=1}^{n+3}\left(2^{n+3}-2^{k-1}\right) \\
&=\frac{\left(5 \cdot 4^{n}+9 \cdot 2^{n+1}+33\right) \prod_{k=1}^{n+3}\left(2^{n+3}-2^{k-1}\right)}{12(n+1) !} .
\end{aligned}
$$

When $n=2$, similarly we have $E\left(P_{3} \times \Delta^{2}\right)=12846400$.

When $n=1, \Delta^{n}$ is the interval $I$. $\operatorname{Aut}\left(\mathcal{F}\left(P_{3} \times I\right)\right)$ is isomorphic to $S_{3} \times$ $\mathbb{Z}_{2} \times \mathbb{Z}_{2} \times \mathbb{Z}_{2}$. If $g$ is the generator $a$ of the first $\mathbb{Z}_{2}$-subgroup of $\operatorname{Aut}\left(\mathcal{F}\left(P_{3} \times I\right)\right)$ and $\lambda \in \Lambda_{g}$, then $\lambda\left(s_{1}\right)=\lambda\left(s_{2}\right)$. By the argument of Lemma 3.1, we have $\left|\Lambda_{g}\right|=20 \prod_{k=1}^{4}\left(2^{4}-2^{k-1}\right)$. If $g$ is the generator $b$ of the second $\mathbb{Z}_{2-}$ subgroup of $\operatorname{Aut}\left(\mathcal{F}\left(P_{3} \times I\right)\right)$ and $\lambda \in \Lambda_{g}$, then $\lambda\left(b_{1}\right)=\lambda\left(b_{2}\right)$. By the argument of (I) of Lemma 3.1, we also have $\left|\Lambda_{g}\right|=20 \prod_{k=1}^{4}\left(2^{4}-2^{k-1}\right)$. If $g$ is the automorphism $a b$ of $\operatorname{Aut}\left(\mathcal{F}\left(P_{3} \times I\right)\right)$, similarly we have $\left|\Lambda_{g}\right|=$ $4 \prod_{k=1}^{4}\left(2^{4}-2^{k-1}\right)$. If $g$ is other automorphism and isn't unit element of $\operatorname{Aut}\left(\mathcal{F}\left(P_{3} \times I\right)\right)$, by the nonsingularity condition of characteristic functions, 
we have that $\Lambda_{g}$ is empty. Thus, from Lemma 3.1, we have

$$
E\left(P_{3} \times I\right)=\frac{1}{48}(20+20+4+69) \prod_{k=1}^{4}\left(2^{4}-2^{k-1}\right)=47460 .
$$

In the similar way, we have that there are 98 equivariant homeomorphism classes of small covers over $P_{3}$.

\section{The number of orientable small covers over $P_{3} \times \Delta^{n}$}

NAKAYAma and Nishimura [15] found an orientability condition for a small cover.

Theorem 4.1. For a basis $\left\{e_{1}, \ldots, e_{n}\right\}$ of $\left(\mathbb{Z}_{2}\right)^{n}$, a homomorphism $\varepsilon$ : $\left(\mathbb{Z}_{2}\right)^{n} \longrightarrow \mathbb{Z}_{2}=\{0,1\}$ is defined by $\varepsilon\left(e_{i}\right)=1(i=1, \ldots, n)$. A small cover $M(\lambda)$ over a simple convex polytope $P^{n}$ is orientable if and only if there exists a basis $\left\{e_{1}, \ldots, e_{n}\right\}$ of $\left(\mathbb{Z}_{2}\right)^{n}$ such that the image of $\varepsilon \lambda$ is $\{1\}$.

We call a $\left(\mathbb{Z}_{2}\right)^{n}$-coloring which satisfies the orientability condition in Theorem 4.1 an orientable coloring of $P^{n}$. We can know the existence of orientable small covers over $P_{3} \times \Delta^{n}$ by the existence of orientable colorings and determine the number of equivariant homeomorphism classes.

By $O\left(P^{n}\right)$ we denote the set of all orientable colorings on $P^{n}$. There is a natural action of $G L\left(n, \mathbb{Z}_{2}\right)$ on $O\left(P^{n}\right)$ defined by the correspondence $\lambda \longmapsto \sigma \circ \lambda$, and the action on $O\left(P^{n}\right)$ is free. Assume that $F_{1}, \ldots, F_{n}$ of $\mathcal{F}\left(P^{n}\right)$ meet at one vertex $p$ of $P^{n}$. Let $e_{1}, \ldots, e_{n}$ be the standard basis of $\left(\mathbb{Z}_{2}\right)^{n}$. Write $B\left(P^{n}\right)=\left\{\lambda \in O\left(P^{n}\right) \mid \lambda\left(F_{i}\right)=e_{i}\right.$ for $\left.i=1, \ldots, n\right\}$. It is easy to check that $B\left(P^{n}\right)$ is the orbit space of $O\left(P^{n}\right)$ under the action of $G L\left(n, \mathbb{Z}_{2}\right)$.

Remark 4.2. In fact, we have $B\left(P^{n}\right)=\left\{\lambda \in O\left(P^{n}\right) \mid \lambda\left(F_{i}\right)=e_{i}\right.$ for $i=1, \ldots, n$, and for $n+1 \leq j \leq \ell, \lambda\left(F_{j}\right)=e_{j_{1}}+e_{j_{2}}+\ldots+e_{j_{2 h_{j}+1}}$, where $\left.1 \leq j_{1}<j_{2}<\ldots<j_{2 h_{j}+1} \leq n\right\}$. Below we show that $\lambda\left(F_{j}\right)=$ $e_{j_{1}}+e_{j_{2}}+\ldots+e_{j_{2 h_{j}+1}}$ for $n+1 \leq j \leq \ell$. If $\lambda \in O\left(P^{n}\right)$, there exists a basis $\left\{e_{1}^{\prime}, \ldots, e_{n}^{\prime}\right\}$ of $\left(\mathbb{Z}_{2}\right)^{n}$ such that for $1 \leq i \leq \ell, \lambda\left(F_{i}\right)=e_{i_{1}}^{\prime}+\ldots+e_{i_{2 f_{i}+1}}^{\prime}$, where $1 \leq i_{1}<\ldots<i_{2 f_{i}+1} \leq n$. Since $\lambda\left(F_{i}\right)=e_{i}$ for $i=1, \ldots, n$, we have $e_{i}=e_{i_{1}}^{\prime}+\ldots+e_{i_{2 f_{i}+1}}^{\prime}$. So we obtain that for $n+1 \leq j \leq \ell$, there aren't $j_{1}, \ldots, j_{2 k}$ such that $\lambda\left(F_{j}\right)=e_{j_{1}}+\ldots+e_{j_{2 k}}$, where $1 \leq j_{1}<\ldots<j_{2 k} \leq n$.

Since $B\left(P^{n}\right)$ is the orbit space of $O\left(P^{n}\right)$, we have 
Lemma 4.3. $\left|O\left(P^{n}\right)\right|=\left|B\left(P^{n}\right)\right| \times\left|G L\left(n, \mathbb{Z}_{2}\right)\right|$.

One can define the right action of $\operatorname{Aut}\left(\mathcal{F}\left(P^{n}\right)\right)$ on $O\left(P^{n}\right)$ by $\lambda \times h \longmapsto$ $\lambda \circ h$, where $\lambda \in O\left(P^{n}\right)$ and $h \in \operatorname{Aut}\left(\mathcal{F}\left(P^{n}\right)\right)$. By improving the classifying result on small covers in [13], we have

Theorem 4.4. Two orientable small covers over an n-dimensional simple convex polytope $P^{n}$ are equivariantly homeomorphic if and only if there is $h \in \operatorname{Aut}\left(\mathcal{F}\left(P^{n}\right)\right)$ such that $\lambda_{1}=\lambda_{2} \circ h$, where $\lambda_{1}$ and $\lambda_{2}$ are their corresponding orientable colorings on $P^{n}$.

Proof. We know Theorem 4.4 is true by combining Lemma 5.4 in [13] with Theorem 4.1.

By Theorem 4.4, the number of orbits of $O\left(P^{n}\right)$ under the action of $\operatorname{Aut}\left(\mathcal{F}\left(P^{n}\right)\right)$ is the number of equivariant homeomorphism classes of orientable small covers over $P^{n}$. So we also are going to count the orbits.

Lemma 4.5. When $n \geq 1$, the number of orientable colorings on $P_{3} \times$ $\Delta^{n}$ is

$$
\left|O\left(P_{3} \times \Delta^{n}\right)\right|= \begin{cases}\left(5 \cdot 4^{n-1}+3\right) \prod_{k=1}^{n+3}\left(2^{n+3}-2^{k-1}\right), & n \text { odd } \\ \left(2^{n}+3\right) \prod_{k=1}^{n+3}\left(2^{n+3}-2^{k-1}\right), & n \text { even } .\end{cases}
$$

Proof. Let $e_{1}, e_{2}, \ldots, e_{n+3}$ be the standard basis of $\left(\mathbb{Z}_{2}\right)^{n+3}$. Then $B\left(P_{3} \times \Delta^{n}\right)=\left\{\lambda \in O\left(P_{3} \times \Delta^{n}\right) \mid \lambda\left(s_{1}\right)=e_{1}, \lambda\left(a_{1}\right)=e_{2}, \lambda\left(a_{2}\right)=e_{3}, \lambda\left(b_{i}\right)=\right.$ $\left.e_{i+3}, 1 \leq i \leq n\right\}$. Then, by Lemma 4.3, we have that $\left|O\left(P_{3} \times \Delta^{n}\right)\right|=$ $\left|B\left(P_{3} \times \Delta^{n}\right)\right| \times\left|G L\left(n+3, \mathbb{Z}_{2}\right)\right|=\prod_{k=1}^{n+3}\left(2^{n+3}-2^{k-1}\right)\left|B\left(P_{3} \times \Delta^{n}\right)\right|$. The calculation of $\left|B\left(P_{3} \times \Delta^{n}\right)\right|$ is divided into two cases: (I) $n$ odd, (II) $n$ even.

(I) $n$ odd

Write:

$$
\begin{aligned}
B_{0}\left(P_{3} \times \Delta^{n}\right) & =\left\{\lambda \in B\left(P_{3} \times \Delta^{n}\right) \mid \lambda\left(b_{n+1}\right)=e_{4}+\ldots+e_{n+3}\right\} \\
B_{1}\left(P_{3} \times \Delta^{n}\right) & =\left\{\lambda \in B\left(P_{3} \times \Delta^{n}\right) \mid \lambda\left(b_{n+1}\right)=e_{4}+\ldots+e_{n+3}+e_{k_{1}}+e_{k_{2}},\right. \\
1 & \left.\leq k_{1}<k_{2} \leq 3\right\} .
\end{aligned}
$$

By the definition of $B\left(P^{n}\right)$, we have that $\left|B\left(P_{3} \times \Delta^{n}\right)\right|=\left|B_{0}\left(P_{3} \times \Delta^{n}\right)\right|+$ $\left|B_{1}\left(P_{3} \times \Delta^{n}\right)\right|$. Then, our argument proceeds as follows. 
Case 1. Calculation of $\left|B_{0}\left(P_{3} \times \Delta^{n}\right)\right|$.

In this case, $\lambda\left(a_{3}\right)=e_{2}+e_{3}+e_{m_{1}} \ldots+e_{m_{l}}$, where $1 \leq m_{1}<\ldots<m_{l} \leq$ $n+3, m_{1} \neq 2, m_{1} \neq 3, \ldots, m_{l} \neq 2, m_{l} \neq 3, l$ odd and $1 \leq l \leq n+1$. When $\lambda\left(a_{3}\right)=e_{2}+e_{3}+e_{m_{1}}+\ldots+e_{m_{l}}, 4 \leq m_{1}<\ldots<m_{l} \leq n+3, l$ odd and $1 \leq l \leq n, \lambda\left(s_{2}\right)=e_{1}+e_{n_{1}}+\ldots+e_{n_{j}}, 2 \leq n_{1}<\ldots<n_{j} \leq n+3, j$ even and $0 \leq j \leq n+2$. When $\lambda\left(a_{3}\right)=e_{2}+e_{3}+e_{1}+e_{m_{1}}+\ldots+e_{m_{l}}, 4 \leq m_{1}<\ldots<$ $m_{l} \leq n+3, l$ even and $0 \leq l \leq n, \lambda\left(s_{2}\right)=e_{1}+e_{g_{1}}+\ldots+e_{g_{k}}, 4 \leq g_{1}<\ldots<$ $g_{k} \leq n+3, k$ even and $0 \leq k \leq n$. Thus, we have $\left|B_{0}\left(P_{3} \times \Delta^{n}\right)\right|=5 \cdot 4^{n-1}$.

Case 2. Calculation of $\left|B_{1}\left(P_{3} \times \Delta^{n}\right)\right|$.

In this case, no matter which value of $\lambda\left(b_{n+1}\right)$ is chosen, we have $\lambda\left(a_{3}\right)=$ $e_{2}+e_{3}+e_{1}, \lambda\left(s_{2}\right)=e_{1}$. Thus, we have $\left|B_{1}\left(P_{3} \times \Delta^{n}\right)\right|=3$.

(II) $n$ even

Write

$$
\begin{aligned}
& B_{0}^{\prime}\left(P_{3} \times \Delta^{n}\right)=\left\{\lambda \in B\left(P_{3} \times \Delta^{n}\right) \mid \lambda\left(b_{n+1}\right)=e_{4}+\ldots+e_{n+3}+e_{h_{1}},\right. \\
& \left.1 \leq h_{1} \leq 3\right\}, \\
& B_{1}^{\prime}\left(P_{3} \times \Delta^{n}\right)=\left\{\lambda \in B\left(P_{3} \times \Delta^{n}\right) \mid \lambda\left(b_{n+1}\right)=e_{4}+\ldots+e_{n+3}+e_{1}+e_{2}+e_{3}\right\} .
\end{aligned}
$$

By the definition of $B\left(P^{n}\right)$, we have that $\left|B\left(P_{3} \times \Delta^{n}\right)\right|=\mid B_{0}^{\prime}\left(P_{3} \times\right.$ $\left.\Delta^{n}\right)|+| B_{1}^{\prime}\left(P_{3} \times \Delta^{n}\right) \mid$. Then, our argument proceeds as follows.

Case 1. Calculation of $\left|B_{0}^{\prime}\left(P_{3} \times \Delta^{n}\right)\right|$. The argument is divided into two cases:

$$
\lambda\left(b_{n+1}\right)=e_{4}+\ldots+e_{n+3}+e_{1} .
$$

In this case, $\lambda\left(a_{3}\right)=e_{2}+e_{3}+e_{m_{1}} \ldots+e_{m_{l}}$, where $1 \leq m_{1}<\ldots<m_{l} \leq n+$ $3, m_{1} \neq 2, m_{1} \neq 3, \ldots, m_{l} \neq 2, m_{l} \neq 3, l$ odd and $1 \leq l \leq n+1, \lambda\left(s_{2}\right)=e_{1}$.

$$
\lambda\left(b_{n+1}\right)=e_{4}+\ldots+e_{n+3}+e_{2} \text { or } e_{4}+\ldots+e_{n+3}+e_{3} .
$$

In this case, no matter which value of $\lambda\left(b_{n+1}\right)$ is chosen, we have $\lambda\left(a_{3}\right)=$ $e_{2}+e_{3}+e_{1}, \lambda\left(s_{2}\right)=e_{1}$. Thus, we have $\left|B_{0}^{\prime}\left(P_{3} \times \Delta^{n}\right)\right|=2^{n}+2$.

Case 2. Calculation of $\left|B_{1}^{\prime}\left(P_{3} \times \Delta^{n}\right)\right|$. In this case, $\lambda\left(a_{3}\right)=e_{2}+e_{3}+e_{1}$, $\lambda\left(s_{2}\right)=e_{1}$. Thus, we have $\left|B_{1}^{\prime}\left(P_{3} \times \Delta^{n}\right)\right|=1$. 
The proof of Theorem 1.2. When $n \geq 3$, from Theorem 4.4, Burnside Lemma and Lemma 2.5, we have that

$$
E_{o}\left(P_{3} \times \Delta^{n}\right)=\frac{1}{12(n+1) !} \sum_{g \in \operatorname{Aut}\left(\mathcal{F}\left(P_{3} \times \Delta^{n}\right)\right)}\left|\Lambda_{g}\right|,
$$

where $\Lambda_{g}=\left\{\lambda \in O\left(P_{3} \times \Delta^{n}\right) \mid \lambda=\lambda \circ g\right\}$.

When $n \geq 3$ and $n$ is odd, the automorphism group $\operatorname{Aut}\left(\mathcal{F}\left(P_{3} \times \Delta^{n}\right)\right)$ is isomorphic to $S_{3} \times \mathbb{Z}_{2} \times S_{n+1}$. If $g$ is the generator of the $\mathbb{Z}_{2}$-subgroup of $\operatorname{Aut}\left(\mathcal{F}\left(P_{3} \times \Delta^{n}\right)\right)$ and $\lambda \in \Lambda_{g}$, then $\lambda\left(s_{1}\right)=\lambda\left(s_{2}\right)$. By the argument of (I) of Lemma 4.5, we have $\left|\Lambda_{g}\right|=\left(2^{n}+3\right) \prod_{k=1}^{n+3}\left(2^{n+3}-2^{k-1}\right)$. If $g$ is other automorphism and isn't unit element of $\operatorname{Aut}\left(\mathcal{F}\left(P_{3} \times \Delta^{n}\right)\right)$, then $\Lambda_{g}$ is empty. Thus, from Lemma 4.5, when $n \geq 3$ and $n$ is odd,

$$
\begin{aligned}
E_{o}\left(P_{3} \times \Delta^{n}\right) & =\frac{1}{12(n+1) !}\left(2^{n}+3+5 \cdot 4^{n-1}+3\right) \prod_{k=1}^{n+3}\left(2^{n+3}-2^{k-1}\right) \\
& =\frac{\left(5 \cdot 4^{n-1}+2^{n}+6\right) \prod_{k=1}^{n+3}\left(2^{n+3}-2^{k-1}\right)}{12(n+1) !} .
\end{aligned}
$$

When $n \geq 4$ and $n$ is even, using the above method we have

$$
E_{o}\left(P_{3} \times \Delta_{n}\right)=\frac{\left(2^{n+1}+6\right) \prod_{k=1}^{n+3}\left(2^{n+3}-2^{k-1}\right)}{12(n+1) !} .
$$

When $n=2$, similarly we have $E_{o}\left(P_{3} \times \Delta_{2}\right)=972160$.

When $n=1, \operatorname{Aut}\left(\mathcal{F}\left(P_{3} \times \Delta^{n}\right)\right)$ is isomorphic to $S_{3} \times \mathbb{Z}_{2} \times \mathbb{Z}_{2} \times \mathbb{Z}_{2}$. If $g$ is the generator $a$ of the first $\mathbb{Z}_{2}$-subgroup of $\operatorname{Aut}\left(\mathcal{F}\left(P_{3} \times I\right)\right)$ and $\lambda \in$ $\Lambda_{g}$, then $\lambda\left(s_{1}\right)=\lambda\left(s_{2}\right)$. By the argument of (I) of Lemma 4.5, we have $\left|\Lambda_{g}\right|=5 \prod_{k=1}^{4}\left(2^{4}-2^{k-1}\right)$. If $g$ is the generator $b$ of the second $\mathbb{Z}_{2}$-subgroup of $\operatorname{Aut}\left(\mathcal{F}\left(P_{3} \times I\right)\right)$ and $\lambda \in \Lambda_{g}$, then $\lambda\left(b_{1}\right)=\lambda\left(b_{2}\right)$. By the argument of Case 1 in (I) of Lemma 4.5, we also have $\left|\Lambda_{g}\right|=5 \prod_{k=1}^{4}\left(2^{4}-2^{k-1}\right)$. If $g$ is the automorphism $a b$ of $\operatorname{Aut}\left(\mathcal{F}\left(P_{3} \times I\right)\right)$, similarly we have $\left|\Lambda_{g}\right|=$ $2 \prod_{k=1}^{4}\left(2^{4}-2^{k-1}\right)$. If $g$ is other automorphism and isn't unit element of $\operatorname{Aut}\left(\mathcal{F}\left(P_{3} \times I\right)\right)$, then $\Lambda_{g}$ is empty. Thus, from Lemma 4.5, we have

$$
E_{o}\left(P_{3} \times I\right)=\frac{1}{48}(5+5+2+8) \prod_{k=1}^{4}\left(2^{4}-2^{k-1}\right)=8400 .
$$

In the similar way, we have that there are 28 equivariant homeomorphism classes of orientable small covers over $P_{3}$. 
Acknowledgments. This work is supported by the National Natural Science Foundation of China (No. 11201126 and 11371018), SRFDP (No. 20121303110004), the Basic Science and Technological Frontier Project of Henan (No. 122300410414 and No. 132300410432) and the research program for scientific technology of Henan province (No. 13A110540).

Finally, the author would like to thank the referee for the invaluable comments.

\section{REFERENCES}

1. Alperin, J.L.; Bell, R.B.- Groups and Representations, Graduate Texts in Mathematics, 162, Springer-Verlag, New York, 1995

2. Appel, K.; Haken, W. - Every planar map is four colorable. I. Discharging, Illinois J. Math., 21 (1977), 429-490.

3. CAI, M.; Chen, X.; LÜ, Z. - Small covers over prisms, Topology Appl., 154 (2007), $2228-2234$.

4. CAO, X.; Lü, Z. - Cohomological rigidity and the number of homeomorphism types for small covers over prisms, Topology Appl., 158 (2011), 813-834.

5. Chen, Y.; WAng, Y. - Orientable small covers over products of a prism with a simplex, An. Ştiinţ. Univ. "Ovidius" Constanţa, Ser. Mat., 19 (2011), 71-84.

6. CHоi, S. - The number of small covers over cubes, Algebr. Geom. Topol., 8 (2008), $2391-2399$

7. Choi, S. - The number of orientable small covers over cubes, Proc. Japan Acad. Ser. A Math. Sci., 86 (2010), 97-100.

8. Choi, S.; Panov, T.; Suh, D.Y. - Toric cohomological rigidity of simple convex polytopes, J. Lond. Math. Soc., 82 (2010), 343-360.

9. Davis, M.W.; Januszkiewicz, T. - Convex polytopes, Coxeter orbifolds and torus actions, Duke Math. J., 62 (1991), 417-451.

10. Garrison, A.; Scott, R. - Small covers of the dodecahedron and the 120-cell, Proc. Amer. Math. Soc., 131 (2003), 963-971.

11. Kuroki, S. - Operations on 3-dimensional small covers, Chin. Ann. Math. Ser. B, 31 (2010), 393-410.

12. Lü, Z. - 2-torus manifolds, cobordism and small covers, Pacific J. Math., 241 (2009), $285-308$.

13. Lü, Z.; Masuda, M. - Equivariant classification of 2-torus manifolds, Colloq. Math., 115 (2009), 171-188. 
14. Lü, Z.; Yu, L. - Topological types of 3-dimensional small covers, Forum Math., 23 (2011), 245-284.

15. NAKAyAma, H.; Nishimura, Y. - The orientability of small covers and coloring simple polytopes, Osaka J. Math., 42 (2005), 243-256.

16. Nishimura, Y. - Combinatorial constructions of three-dimensional small covers, $\mathrm{Pa-}$ cific J. Math., 256 (2012), 177-199.

17. Wang, Y.; Chen, Y. - Small covers over products of a polygon with a simplex, Turkish J. Math., 36 (2012), 161-172.

18. Ziegler, G.M. - Lectures on Polytopes, Graduate Texts in Mathematics, 152, Springer-Verlag, New York, 1995.

Received: 9.I.2012

Revised: 14.VI.2012

Accepted: 3.IX.2012
College of Mathematics and Information Science, Henan Normal University, Xinxiang 453007,

P.R. CHINA

cyc810707@163.com 